\title{
DIRAC QUANTUM FIELDS ON A MANIFOLD'
}

BY

J. DIMOCK

\begin{abstract}
On globally hyperbolic Lorentzian manifolds we construct field operators which satisfy the Dirac equation and have a causal anticommutator. Ambiguities in the construction are removed by formulating the theory in terms of $C^{*}$ algebras of local observables. A generalized form of the Haag-Kastler axioms is verified.
\end{abstract}

Introduction. A Lorentzian manifold is a four dimensional manifold $M$ together with a pseudo-Riemannian metric $g$ of signature $(+,-,-,-)$. Such manifolds are widely used as models for space-time. In particular in the absence of boundaries and gravitational fields one uses $M=R^{4}$ and $g=\eta \equiv$ Minkowski metric which has components $\left\{\eta_{a b}\right\}=\operatorname{diag}(1,-1,-1,-1)$.

We are interested in formulating quantum field theories on general Lorentzian manifolds. This means giving up many ideas familiar from the usual Minkowski space treatments. Thus there are no Poincare transformations on $M$, no vacuum, no particle states, and so forth. All one is left with are the field equations. These are to be solved taking as data a representation of the CCR or CAR over a space-like hypersurface $(\mathrm{CCR}=$ canonical commutation relations, $\mathrm{CAR}=$ canonical anticommutation relations). For linear equations this quantum problem can be reduced to a corresponding classical problem for which solutions can be constructed in favorable cases. In the nonlinear case very singular formal solutions can be exhibited which one might hope to make rigorous.

The procedure sketched above is fraught with ambiguities. What hypersurface should one take? what representation of the CCR/CAR? and so forth. In general there is no natural choice and one should show that all choices lead to the same theory. To do this in a fundamental way it seems necessary to abandon the field operators themselves and instead consider the $C^{*}$ algebras that they generate. This algebraic approach was originally formulated for Minkowski space and seems all the more appropriate here.

In the Haag-Kastler approach [10] one focuses on the $C^{*}$ algebras associated with local regions of space-time-the algebras of local observables. The original formulation on Minkowski space consisted of a list of structural properties or axioms which such algebras ought to obey. In a recent paper [6] we have proposed a generalization of these axioms to Lorentzian manifolds. The scalar field was given as an example.

Received by the editors July 23, 1980 and, in revised form, December 11, 1980.

1980 Mathematics Subject Classification. Primary 81A17.

${ }^{1}$ Supported by National Science Foundation, PHY77-21740. 
The present paper continues this work and studies Dirac quantum fields in this algebraic setting. The contents of the paper are as follows:

§I. The Dirac equation. We develop the mathematical concepts of spin structures, spin connections, etc., which are needed to formulate the classical Dirac equation. Our treatment roughly follows the original work of Lichnerowicz [16].

§II. Classical solutions. For globally hyperbolic manifolds we construct fundamental solutions for the Dirac operator using a general theorem of Leray [18]. We also show that the global Cauchy problem is well posed.

§III. Quantum solutions. Starting with a representation of the CAR over a Cauchy hypersurface and using the fundamental solutions we construct quantum field operators which solve the Dirac equation and have a causal anticommutator. This construction is similar to the one given for the scalar quantum field by Isham [13] and Dimock [6].

$\S I V$. Local algebras. From the fields we construct $C^{*}$ algebras of local observables. These structures are shown to be independent of the representation of the CAR, the Cauchy surface, and the choice of spin structure. The equivalences are conveniently formulated in terms of category theory. Finally the generalized Haag-Kastler axioms are verified.

\section{The Dirac equation.}

A. Spinors. We review the geometric setting for the Dirac equation (see [4], [16]). Our starting point is a representation of the Dirac-Clifford algebra as given by $4 \times 4$ matrices $\gamma_{0}, \ldots, \gamma_{3}$ satisfying

$$
\gamma_{a} \gamma_{b}+\gamma_{b} \gamma_{a}=2 \eta_{a b} I
$$

Given any two such representations $\gamma_{a}, \gamma_{a}^{\prime}$ there is a nonsingular matrix $M$ (unique up to a multiple of the identity) such that $\gamma_{a}^{\prime}=M \gamma_{a} M^{-1}$ [19]. We generally work with a fixed but arbitrary representation. The $\gamma_{a}$ are used to define a covering group for the Lorentz group as we explain next, and later to construct a differential operator whose square is a wave operator.

Given $\gamma_{a}$ we consider the Lie group Spin consisting of all matrices $S$ satisfying $\operatorname{det} S=1$ and

$$
S \gamma_{a} S^{-1}=\gamma_{b} \Lambda_{a}^{b}
$$

for some real numbers $\Lambda_{a}^{b}$. From (1.1) we find that $\eta_{c d} \Lambda_{a}^{c} \Lambda_{b}^{d}=\eta_{a b}$ which says that the matrix $\Lambda=\left\{\Lambda_{a}^{b}\right\}$ belongs to the Lorentz group $\mathcal{L}$. The map $S \rightarrow \Lambda(S)$ is a 2-1 homomorphism from Spin onto $\mathcal{L}$. In the following we restrict attention to $\operatorname{Spin}_{0}$, the connected component of the identity in Spin, and its image $\mathscr{L}_{0}$ which is the connected component of the identity in $\mathcal{L}$. ( $\varrho_{0}$ is of ten written $\mathcal{L}_{+}^{\uparrow}$, and $\operatorname{Spin}_{0}$ is isomorphic to $\operatorname{SL}(2, C)$.)

Let $(M, g)$ be an oriented time-oriented Lorentzian manifold, and let $F M$ be the bundle of oriented time-oriented orthonormal frames in the tangent space $T M$. An element $\xi$ of $F M$ consists of four tangent vectors $\left(\xi_{0}, \ldots, \xi_{3}\right)$ at a point in $M$ which are oriented, which have $\xi_{0}$ in the forward cone, and which satisfy $g\left(\xi_{a}, \xi_{b}\right)=\eta_{a b}$. There is a right action of $\mathcal{E}_{0}$ on $F M$ defined by $\left(R_{\Lambda} \xi\right)_{a}=\xi_{b} \Lambda_{a}^{b}$, and with this choice $F M$ is a principal $\varrho_{0}$ bundle over $M$. 
A spin structure for $(M, g)$ is a pair $(S M, p)$ consisting of a principal $\operatorname{Spin}_{0}$ bundle $S M$ over $M$ (the spin frame bundle) and a bundle homomorphism $p$ : $S M \rightarrow F M$ which preserves the base point and which satisfies $p \circ R_{S}=R_{\Lambda(S)}{ }^{\circ} p$ where $R_{S}$ is the right action of $\operatorname{Spin}_{0}$ on $S M$. Spin structures are not unique. Spin sructures $\left(S_{1} M, p_{1}\right)$ and $\left(S_{2} M, p_{2}\right)$ are said to be equivalent if there is a $\operatorname{Spin}_{0}$-bundle isomorphism $\tau: S_{1} M \rightarrow S_{2} M$ preserving the base point such that $p_{1}=p_{2} \circ \tau$.

The existence of spin structures depends on the topology of the manifold (e.g. [1], [17]). Spin structures exist if and only if the second Stieffel-Whitney class for $M$ vanishes. We make even stronger assumptions later on. Equivalence classes of spin structures may be labeled by elements of the cohomology group $H^{1}\left(M, Z_{2}\right)$. If this is nontrivial one speaks of exotic spin structures. The possible physical significance of exotic spin structures has been recently discussed in the literature (e.g. [5], [20]).

The Dirac spinor bundle is now the associated vector bundle $D M=$ $\left(S M \times C^{4}\right) / S$. That is $D M$ is the orbit space for the action $R_{S} \times S^{-1}$ of $S_{p i n}$ on $S M \times C^{4}$. A spinor field is a cross-section of $D M$, i.e. a smooth map $u: M \rightarrow D M$ such that $u(x) \in D M_{x}$ where $D M_{x}$ is the fiber over $x$. The space of all spinor fields is denoted $C^{\infty}(D M)$.

We also introduce the dual vector bundle $D^{*} M=\cup_{x} D^{*} M_{x}$ where $D^{*} M_{x}$ is the dual vector space to $D M_{x}$. A cospinor field $v$ is a cross-section of $D^{*} M$, and the space of all such cross-sections is denoted $C^{\infty}\left(D^{*} M\right)$. If $u \in C^{\infty}(D M)$ and $v \in C^{\infty}\left(D^{*} M\right)$ we may form a function $v u=v(u) \in C^{\infty}(M)$ by $(v u)(x)=$ $v(x)(u(x))$.

A local cross-section $E: U \subset M \rightarrow S M$ determines a moving frame $\left(E_{1}, \ldots, E_{4}\right)$ in $D M$. Each $E_{A}$ is a local cross-section $E_{A}: U \rightarrow D M$ defined by $E_{A}(x)=$ $i\left(E(x), \omega_{A}\right)$ where $\omega_{1}, \ldots, \omega_{4}$ is the standard basis for $\mathbf{C}^{4}$ and $i: S M \times C^{4} \rightarrow D M$ is the canonical injection. Any $u \in C^{\infty}(D M)$ can be expressed as $u=u^{A} E_{A}$ (summation convention) where $u^{A} \in C^{\infty}(M)$. If $E^{\prime}$ is another cross-section we have $E^{\prime}=R_{S^{-1}} E$ for some $S: U \cap U^{\prime} \rightarrow \operatorname{Spin}_{0}$. Then the frames are related by $E_{A}^{\prime}=E_{B}\left(S^{-1}\right)_{A}^{B}$ and the components by $u^{\prime A}=S_{B}^{A} u^{B}$.

By taking tensor products of $D M, D^{*} M, T M, T^{*} M$ we may form a mixed spinor-tensor algebra. A cross-section $E$ of $S M$ determines moving frames in each of these bundles. We have $\left(E_{A}\right)$ in $D M$ as defined above, the dual frame $\left(E^{B}\right)$ in $D^{*} M$ satisfying $E^{B}\left(E_{A}\right)=\delta_{A}^{B}$, the orthonormal frame $\left(e_{a}\right)=e=p \circ E$ in $T M$, and the dual frame $\left(e^{b}\right)$ in $T^{*} M$ satisfying $e^{b}\left(e_{a}\right)=\delta_{a}^{b}$. Under a change of section given by $S: U \cap U^{\prime} \rightarrow \operatorname{Spin}_{0}$ we have the change of frames

$$
\begin{array}{cl}
E_{A}^{\prime}=E_{B}\left(S^{-1}\right)_{A}^{B}, & \left(E^{\prime}\right)^{B}=S_{A}^{B} E^{A}, \\
e_{a}^{\prime}=e_{b}\left(\Lambda\left(S^{-1}\right)\right)_{a}^{b}, & \left(e^{\prime}\right)^{b}=(\Lambda(S))_{a}^{b} e^{a}
\end{array}
$$

and corresponding changes in the components of any spinor-tensor. For example if $f \in C^{\infty}\left(T^{*} M \otimes D M \otimes D^{*} M\right)$ is expressed in one frame as $f=f_{a B}^{A} e^{a} \otimes E_{A} \otimes$ $E^{B}$, then the components in the second frame are given by

$$
\left(f^{\prime}\right)_{a^{\prime} B^{\prime}}^{A^{\prime}}=\left(\Lambda\left(S^{-1}\right)\right)_{a^{\prime}}^{a} S_{A}^{A^{\prime}}\left(S^{-1}\right)_{B^{\prime}}^{B} f_{a B^{\prime}}^{A}
$$

Tensor indices may be raised or lowered using $\eta_{a b}=\eta^{a b}$. 
In the following we will be particularly interested in the spinor-tensor $\gamma$ which has components $\gamma_{a B}^{A}=\left(\gamma_{a}\right)_{B}^{A}$ in any frame (use equation (1.2)).

We also note that we can contract spinor-tensors by summing over dual indices. For example $v u$ is expressed as $v^{A} u_{A}$. If $a$ is a vector field we define $a$ to be the contraction with $\gamma$, so that $a_{B}^{A}=a^{a} \gamma_{a B}^{A}$. For a spinor $u$, $(a u)$ is the spinor with components $(a u)^{A}=(a)_{B}^{A} u^{B}$. For a cospinor $v$, $a v$ has components $(a v)_{B}=v_{A}(a)_{B}^{A}$. Finally if $v u$ has compact support we may define $\langle v, u\rangle=\int_{M} v u d V$ where $d V$ is the volume element determined by $g$.

B. Adjoint spinors. The adjoint matrices $\gamma_{a}^{*}$ also satisfy (1.1). Thus we have $\gamma_{a}^{*}=\beta \gamma_{a} \beta^{-1}$ for a unique $\beta$ with det $\beta=1$. We also have

$$
\begin{aligned}
\left(\gamma_{a}\right)^{*} & =\left(S^{-1} \gamma_{b} \Lambda(S)_{a}^{b} S\right)^{*} \\
& =S^{*} \beta \gamma_{b} \beta^{-1} \Lambda(S)_{a}^{b}\left(S^{-1}\right)^{*}=\beta^{\prime} \gamma_{a}\left(\beta^{\prime}\right)^{-1}
\end{aligned}
$$

where $\beta^{\prime}=S^{*} \beta S$. Since also $\operatorname{det} \beta^{\prime}=1$ we have $\beta^{\prime}=\beta$, that is $S^{*} \beta S=\beta$.

Given $u \in C^{\infty}(D M)$ we now define an adjoint spinor $u^{+} \in C^{\infty}\left(D^{*} M\right)$. In a local frame $u=u^{A} E_{A}$ and we set $u_{B}^{+}=\overline{u^{A}} \beta_{A B}$ where $\beta_{A B}$ are the matrix elements of $\beta$. Under a change of frame $\left(u^{\prime}\right)_{B}^{+}=u_{A}^{+}\left(S^{-1}\right)_{B}^{A}$ (since $S^{*} \beta=\beta S^{-1}$ ). Thus $u^{+}=u_{B}^{+} E^{B}$ defines a frame independent covariant spinor. (Note. Our notation $u^{+}=\bar{u} \beta$ differs from the usual physics notation which would be $\bar{u}=u^{+} \beta$.)

Proposition 1.1. If $\mathrm{n}$ is a forward time-like vector field on $M$ then $\left(u_{1}, u_{2}\right)=$ $\left\langle u_{1}^{+}, \mathfrak{x} u_{2}\right\rangle$ is an inner product on $C_{0}^{\infty}(D M)$.

Proof. We must show that $\left\langle u^{+}, \mathfrak{x} u\right\rangle$ is positive definite and it suffices that $u^{+} \mathfrak{X} u$ is positive definite for $u \in D M_{x}$. Going to a particular frame we must show that $(u, \beta \mathbb{\gamma} u)=\left(u, \beta \gamma_{a} u\right) \mathrm{n}^{a}$ is positive definite for $u \in C^{4}$. Here $(\cdot, \cdot)$ is the usual inner product on $C^{4}$, and the conditions on $\mathrm{n}$ are $\mathrm{n}^{a} \mathrm{n}^{b} \eta_{a b}>0$ and $\mathrm{n}^{0}>0$.

First suppose that we have one of the standard representations with $\gamma_{0}^{*}=\gamma_{0}$ and $\gamma_{a}^{*}=-\gamma_{a}, a=1,2,3$, so that $\beta=\gamma_{0}$. We may assume $\mathrm{n}^{a} \mathrm{n}^{b} \eta_{a b}=1$ and choose $\Lambda_{b}^{a} \mathrm{n}^{a}=\delta_{0}^{a}$. If $S \in \operatorname{Spin}_{0}$ satisfies $\Lambda(S)=\Lambda$ then $S x S^{-1}=\gamma_{0}$.

Thus we have $(u, \beta \not u)=\left(u, \beta S^{-1} \gamma_{0} S u\right)=\left(S u, \beta \gamma_{0} S u\right)=(S u, S u) \geqslant 0$ with equality iff $S u=0$, i.e. $u=0$.

Now consider an arbitrary representation $\gamma_{a}^{\prime}=M^{-1} \gamma_{a} M$. Then $\left(\gamma_{a}^{\prime}\right)^{*}=$ $\beta^{\prime} \gamma_{a}^{\prime}\left(\beta^{\prime}\right)^{-1}$ where $\beta^{\prime}=M^{*} \beta M|\operatorname{det} M|^{-2}$. Then

$$
\left(u, \beta^{\prime} \mathbf{x}^{\prime} u\right)=(M u, \beta \mathrm{R} M u)|\operatorname{det} M|^{-2} \geqslant 0
$$

with equality iff $M u=0$, i.e. $u=0$.

C. Connections. The metric $g$ determines a connection on $M$. This can be regarded as a covariant derivative $\nabla: C^{\infty}(T M) \rightarrow C^{\infty}\left(T^{*} M \otimes T M\right)$. If $e$ is a local orthonormal frame we have

$$
\nabla e_{a}=\omega_{a}^{b} \otimes e_{b}=\Gamma_{c a}^{b} e^{c} \otimes e_{b}
$$

which defines connection forms $\omega_{a}^{b}$ and Christoffel symbols $\Gamma_{c a}^{b}$. Alternatively the connection can be regarded as a $\operatorname{Lie}\left(\varrho_{0}\right)$ valued one-form $\omega$ on $F M$ satisfying certain properties. Then $e^{*}(\omega)$ is a $\operatorname{Lie}\left(\varrho_{0}\right)$ valued one-form on $M$ whose matrix elements are just $\omega_{a}^{b}$. 
The spinor connection $\sigma$ is the pull-back of $\omega$ to the spin-frame bundle $S M$. We put $\sigma=\left(\Lambda^{\prime}\right)^{-1} p^{*}(\omega)$ where $\Lambda^{\prime}$ is the isomorphism from $\operatorname{Lie}\left(\operatorname{Spin}_{0}\right)$ to $\operatorname{Lie}\left(\varrho_{0}\right)$. One can check that $\sigma$ is a connection form on $S M$. If $E$ is a local cross-section then $E^{*}(\sigma)$ is a $\operatorname{Lie}\left(\operatorname{Spin}_{0}\right)$ valued one-form on $M$. The matrix elements are one-forms denoted $\sigma_{A}^{B}$. We now define a covariant derivative

$$
\nabla: C^{\infty}(D M) \rightarrow C^{\infty}\left(T^{*} M \otimes D M\right)
$$

by $\nabla E_{A}=\sigma_{A}^{B} \otimes E_{B}=\sigma_{a A}^{B} e^{a} \otimes E_{B}$ which will be independent of $E$. If $e=p \circ E$ then $E^{*}(\sigma)=\left(\Lambda^{\prime}\right)^{-1} e^{*}(\omega)$ which enables one to compute

$$
\sigma_{a A}^{B}=-\frac{1}{4} \Gamma_{a d}^{b} \gamma_{b C}^{B} \gamma_{A}^{d C} \text {. }
$$

If $\nabla_{a} u^{A}$ are the components of $\nabla\left(u^{A} E_{A}\right)=\left(d u^{A}\right) E_{A}+u^{A} \nabla E_{A}$ we have $\nabla_{a} u^{A}=$ $\partial_{a} u^{A}+\sigma_{a B}^{A} u^{B}$ where $\partial_{a} f=d f\left(e_{a}\right)$.

The definition of the covariant derivative can be extended to the full spinortensor algebra in the usual way. The building blocks are $\nabla f=d f$ for functions, together with

$$
\begin{aligned}
\nabla e_{a}=\omega_{a}^{b} \otimes e_{b}, & \nabla e^{b}=-\omega_{a}^{b} \otimes e^{a}, \\
\nabla E_{A}=\sigma_{A}^{B} \otimes E_{B}, & \nabla E^{B}=-\sigma_{A}^{B} \otimes E^{A},
\end{aligned}
$$

and these are pieced together using the Leibniz rule. For example if $f$ is a spinor-tensor with components $f_{a B}^{A}$ as before then the components of $\nabla f$ denoted $\nabla_{b} f_{a B}^{A}$ are $\nabla_{b} f_{a B}^{A}=\partial_{b} f_{a B}^{A}-\Gamma_{b a}^{c} f_{c B}^{A}+\sigma_{b D}^{A} f_{a B}^{D}-f_{a D}^{A} \sigma_{b B}^{D}$. One can show from this that $\nabla \gamma=0$.

D. The Dirac operator. We now define a differential operator $\nabla$ on spinors or cospinors by applying $\nabla$ followed by contraction with $\gamma$. In terms of components $(\nabla u)^{A}=\gamma^{a A}{ }_{B} \nabla_{a} u^{B}$, and $(\nabla v)_{B}=\left(\nabla_{a} v_{A}\right) \gamma^{a A}{ }_{B}$. Thus with spinor indices suppressed we have

$$
\begin{aligned}
& \nabla u=\gamma^{a} \nabla_{a} u=\gamma^{a}\left(\partial_{a} u+\sigma_{a} u\right), \\
& \nabla v=\left(\nabla_{a} v\right) \gamma^{a}=\left(\partial_{a} v-v \sigma_{a}\right) \gamma^{a}
\end{aligned}
$$

Using $\beta \gamma^{a}=\left(\gamma^{a}\right)^{*} \beta$ and $\beta \sigma_{a}=-\sigma_{a}^{*} \beta$ we obtain that $\nabla u^{+}=(\nabla u)^{+}$.

The Dirac equation for spinors $u$ or cospinors $v$ is

$$
(-i \nabla+m) u=0, \quad(i \nabla+m) v=0
$$

where the mass $m$ is any real number. A spinor $u$ is a solution if and only if $u^{+}$is a solution. We immediately note the following important identity [16]

$$
(-i \nabla+m)(i \nabla+m) u=\left(\square-\frac{1}{4} R+m^{2}\right) u
$$

where $\square$ is the spinor wave operator given by

$$
(\square u)^{A}=\eta^{a b} \nabla_{a} \nabla_{b} u^{A} .
$$

In leading order this is just the usual wave operator. 
We also note the following integral identity:

Proposition 1.2. Let $u \in C^{\infty}(D M), v \in C^{\infty}\left(D^{*} M\right)$ and let $D \subset M$ have smooth boundary $\partial D$ with outward normal $n$ and volume element $d S$. If supp $u \cap$ supp $v \cap D$ is compact then

$$
-i \int_{\partial D} v \not x u d S=\int_{D}(v(-i \not \nabla+m) u-((i \not \nabla+m) v) u) d V .
$$

Proof. Since covariant differentiation commutes with contraction, and since $\nabla \gamma=0$ we have $\nabla_{a}\left(v \gamma^{a} u\right)=v(\nabla u)+(\nabla v) u$. Now multiply by $-i$, integrate over $D$, and use Gauss's theorem to obtain the result for $m=0$. The case $m \neq 0$ follows trivially.

E. Distributions. We consider the spaces $C_{0}^{\infty}(D M)$ and $C_{0}^{\infty}\left(D^{*} M\right)$ of sections with compact support. These are given the topology of uniform convergence of all derivatives on a fixed compact set. The section distributions are the continuous linear functionals on these spaces. Any $u \in C^{\infty}(D M)$ defines such a distribution on $h \in C_{0}^{\infty}\left(D^{*} M\right)$ by $u(h)=\langle h, u\rangle$. Furthermore the functional is zero if and only if $u=0$. Thus we have an identification of $C^{\infty}(D M)$ with a subspace of $\left[C_{0}^{\infty}\left(D^{*} M\right)\right]^{\prime}$. Correspondingly we introduce the notation

$$
C^{-\infty}(D M)=\left[C_{0}^{\infty}\left(D^{*} M\right)\right]^{\prime}, \quad C^{-\infty}\left(D^{*} M\right)=\left[C_{0}^{\infty}(D M)\right]^{\prime} .
$$

We give $C^{\infty}(D M)$ and $C^{\infty}\left(D^{*} M\right)$ topologies based on uniform convergence of all derivatives on any compact set. The dual spaces are denoted

$$
C_{0}^{-\infty}(D M)=\left[C^{\infty}\left(D^{*} M\right)\right]^{\prime}, \quad C_{0}^{-\infty}\left(D^{*} M\right)=\left[C^{\infty}(D M)\right]^{\prime} .
$$

These can be shown to consist of the section distributions with compact support.

If $f \in C_{0}^{\infty}(D M), h \in C_{0}^{\infty}\left(D^{*} M\right)$, the identity (1.7) gives

$$
\langle h, \nabla f\rangle=-\langle\nabla h, f\rangle \text {. }
$$

This shows that we may continuously extend $\nabla$ to $C^{-\infty}(D M)$ or $C^{-\infty}\left(D^{*} M\right)$ by defining $\nabla=-(\not)^{\prime}$.

\section{Classical solutions.}

A. Fundamental solutions. To study solutions of the Dirac equation we restrict to space-times $(M, g)$ which are globally hyperbolic in the sense of Leray [3], [18]. This implies that spin structures $(S M, p)$ exist and that $S M$ is a trivial bundle [8], [14] (but not that all structures are equivalent). The global hyperbolicity also gives the existence of fundamental solutions for the Dirac equation due to a general theorem of Leray. Actually the theorem is valid for strictly hyperbolic operators and so does not apply directly to $(-i \nabla+m)$; however by squaring the operator we can reduce the question to the strictly hyperbolic case. The result is:

THEOREM 2.1. (a) $(-i \nabla+m)$ on $C^{\infty}(D M)$ has unique fundamental solutions $S^{ \pm}$: $C_{0}^{\infty}(D M) \rightarrow C^{\infty}(D M)$ satsfying

$$
(-i \not \nabla+m) S^{ \pm}=S^{ \pm}(-i \not \nabla+m)=I \text { on } C_{0}^{\infty}(M), \quad \operatorname{supp}\left(S^{ \pm} f\right) \subset J^{ \pm}(\operatorname{supp} f) .
$$

(b) Similarly $(i \nabla+m)$ on $C^{\infty}\left(D^{*} M\right)$ has unique fundamental solutions $S^{ \pm}$: $C_{0}^{\infty}\left(D^{*} M\right) \rightarrow C^{\infty}\left(D^{*} M\right)$. 
Note. For any $K \subset M, J^{ \pm}(K)$ is the set of all points in $M$ which can be reached by a forward (backward) directed causal curve. A causal curve is a smooth curve whose tangent vectors are either time-like or null.

Proof (CF. [24]). First we show existence of right fundamental solutions. We pick a global spin frame $E$. Then $(-i \not+m)$ can be regarded as the operator on $C^{\infty}\left(M, \mathbf{C}^{4}\right)$ given by (1.3) and it suffices to find $S^{ \pm}: C_{0}^{\infty}\left(M, \mathbf{C}^{4}\right) \rightarrow C^{\infty}\left(M, \mathrm{C}^{4}\right)$ satisfying the stated conditions. Consider the operator $\square-\frac{1}{4} R+m^{2}$ on $C^{\infty}\left(M, C^{4}\right)$ as given by (1.6). In leading order the operator is diagonal and is given by $\eta^{a b} \partial_{a} \partial_{b}$ with respect to the orthonormal frame $e=p \circ E$, or by $g^{a b}\left(\partial^{2} u / \partial x^{a} \partial x^{b}\right)$ with respect to any local coordinate frame. Since $g$ is globally hyperbolic Leray's theorem applies $[18, \S 111]$ and we conclude that there exist $E^{ \pm}: C_{0}^{\infty}\left(M, C^{4}\right) \rightarrow$ $C^{\infty}\left(M, \mathbf{C}^{4}\right)$ which are fundamental solutions for $\square-\frac{1}{4} R+m^{2}$ and have the correct support. Then $S^{ \pm}=(i \nabla+m) E^{ \pm}$satisfies $(-i \nabla+m) S^{ \pm}=I$ and also has the correct support. This supplies $S^{ \pm}$on $C_{0}^{\infty}(D M)$, and $S^{ \pm}$on $C_{0}^{\infty}\left(D^{*} M\right)$ is similar.

To show that the right fundamental solutions are left fundamental solutions we note that, for $h \in C_{0}^{\infty}\left(D^{*} M\right), f \in C_{0}^{\infty}(D M)$ we have

$$
\left\langle S^{ \pm} h, f\right\rangle=\left\langle S^{ \pm} h,(-i \nabla+m) S^{\mp} f\right\rangle=\left\langle h, S^{\mp} f\right\rangle .
$$

Here we use that $\operatorname{supp}\left(S^{ \pm} h\right) \cap \operatorname{supp}\left(S^{\mp} f\right)$ is compact, a consequence of the global hyperbolicity. Then by taking the adjoint of $(i \nabla+m) S^{ \pm}=I$ on $C_{0}^{\infty}\left(D^{*} M\right)$ we obtain $S^{ \pm}(-i \nabla+m)=I$ on $C_{0}^{\infty}(D M)$. Thus $S^{ \pm}$is a left fundamental solution. The uniqueness follows similarly.

REMARK. The operators $S^{ \pm}$on $C_{0}^{\infty}\left(D^{*} M\right)$ have adjoints $\left(S^{ \pm}\right)^{\prime}: C_{0}^{-\infty}(D M) \rightarrow$ $C^{-\infty}(D M)$. Since $\left\langle S^{ \pm} h, f\right\rangle=\left\langle h, S^{\mp} f\right\rangle$ these provide extensions of $S^{\mp}$ to distributions and we have $\left(S^{ \pm}\right)^{\prime}=S^{\mp}$.

We also define the operator $S=S^{+}-S^{-}$on $C_{0}^{\infty}(D M)$ and $C_{0}^{-\infty}(D M)$. This is called the propagator and satisfies $S^{\prime}=-S$.

B. The Cauchy problem. We now construct solutions for the Cauchy problem in three steps: (1) a weak integral identity, (2) a strong existence theorem, and (3) a reformulation of the integral identity. First we need some definitions.

A Cauchy surface $\Sigma$ for $(M, g)$ is a space-like hypersurface such that every endless causal curve intersects it exactly once. The global hyperbolicity implies the existence of $C^{0}$ Cauchy surfaces [9], [11]. We assume that there are also $C^{\infty}$ Cauchy surfaces and consider them exclusively.

Given $\Sigma$ let $D \Sigma$ and $D^{*} \Sigma$ be the vector bundles obtained by restricting $D M$, $D^{*} M$ to $\Sigma$. We let $\rho$ be the restriction operator on the various spaces, e.g. $\rho$ : $C^{\infty}\left(D^{*} M\right) \rightarrow C^{\infty}\left(D^{*} \Sigma\right)$. If $u \in C^{\infty}(D \Sigma), v \in C^{\infty}\left(D^{*} \Sigma\right)$ and $v u$ has compact support we set $\langle v, u\rangle=\int_{\Sigma} v u d S$, where $d S$ is the induced volume element on $\Sigma$. Finally let $\mathrm{n}$ be the forward normal on $\Sigma$.

Proposition 2.2. Let $u \in C^{\infty}(D M)$ satisfy $(-i \not \nabla+m) u=0$, and $\rho u=u_{0} \in$ $C^{\infty}(D \Sigma)$. Then for $h \in C_{0}^{\infty}\left(D^{*} M\right)$,

$$
\langle h, u\rangle=i\left\langle\boldsymbol{x} \rho S h, u_{0}\right\rangle .
$$


Proof. In the identity (1.7) we put $D=\Sigma^{-} \equiv J^{-}(\Sigma)$ and $v=S^{+} h$. The region supp $S^{+} h \cap \Sigma^{-} \subset J^{+}(\operatorname{supp} h) \cap \Sigma^{-}$is compact since $\Sigma$ is a Cauchy surface [11]. Thus we have

$$
\int_{\Sigma^{-}} h u d V=i \int_{\Sigma}\left(S^{+} h\right) \mathrm{x} u_{0}
$$

Similarly with $D=\Sigma^{+}=J^{+}(\Sigma)$ and $v=S^{-} h$ we have

$$
\int_{\Sigma^{+}} h u d V=-i \int_{\Sigma}\left(S^{-} h\right) \times u_{0}
$$

Adding the identities gives the result.

THEOREM 2.3. Let $u_{0} \in C_{0}^{\infty}(D \Sigma)$; then there exists a unique $u \in C^{\infty}(D M)$ so that $(-i \not \nabla+m) u=0$ and $\rho(u)=u_{0}$. Furthermore supp $u \subset J^{+}\left(\operatorname{supp} u_{0}\right) \cup$ $J^{-}\left(\operatorname{supp} u_{0}\right)$.

Proof. The uniqueness follows from the proposition and using the support property for $S$ one can similarly deduce the support. (See Appendix in [6].)

For existence we again choose a global spin frame which trivializes both $D M$ and $D \Sigma$. The problem is then, given $u_{0} \in C_{0}^{\infty}\left(\Sigma, C^{4}\right)$, to find $u \in C^{\infty}\left(M, \mathbf{C}^{4}\right)$ satisfying the equation and $u \mid \Sigma=u_{0}$. Using techniques developed for the Klein-Gordon equation [6], one can prove that the Cauchy problem for $\left(\square-\frac{1}{4} R+m^{2}\right) w=0$ has global solutions. (The proof is an elaboration of the usual construction using energy inequalities). Let $w$ be the solution with $w \mid \Sigma=0$ and $\partial w / \partial n=-i \not x u_{0}$, and let $u=(i \not \nabla+m) w$. Then $(-i \not \nabla+m) u=0$ by (1.5). Note that since $w=0$ on $\Sigma$ we

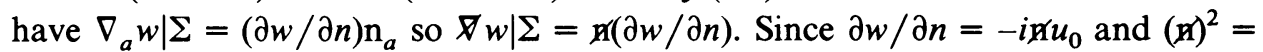
1 we have $\nabla w \mid \Sigma=-i u_{0}$ which gives $u \mid \Sigma=u_{0}$.

REMARK. The operator $\rho$ has an adjoint $\rho^{\prime}: C_{0}^{-\infty}(D \Sigma) \rightarrow C_{0}^{-\infty}(D M)$. Then $S \rho^{\prime}$ maps $C_{0}^{-\infty}(D \Sigma)$ to $C^{-\infty}(D M)$. The next result improves this.

Proposition 2.4. (a) $S \rho^{\prime}$ restricts to a continuous operator from $C_{0}^{\infty}(D \Sigma)$ to $C^{\infty}(D M)$.

(b) The solution of the Cauchy problem with data $u_{0} \in C_{0}^{\infty}(D \Sigma)$ is $u=-i S \rho^{\prime} \not u_{0}$.

(c) $-i \rho S \rho^{\prime} \not x=I$ on $C_{0}^{\infty}(D \Sigma)$.

(d) $-i S \rho^{\prime} \not \rho S=S$ on $C_{0}^{\infty}(D M)$.

Proof. Let $u \in C^{\infty}(D M)$ be the solution of the Cauchy problem with data $u_{0} \in C_{0}^{\infty}(D \Sigma)$. Then Proposition 2.2 says that $u=-i S \rho^{\prime} \mathbf{x} u_{0}$ in the sense of distributions. Since $u_{0}$ is arbitrary this shows that $-i S \rho^{\prime}$ x maps $C_{0}^{\infty}(D \Sigma)$ to $C^{\infty}(D M)$ and hence so does $S \rho^{\prime}$. The continuity follows by the closed graph theorem (cf. [4, p. 391]). This proves (a) and (b). Now (c) follows by applying $\rho$ to the identity in (b). Finally (d) follows by inserting $u_{0}=\rho u$ to get $u=-i S \rho^{\prime}$ x $\rho u$ for any solution $u$ with compact support on $\Sigma$. Taking $u=S f, f \in C_{0}^{\infty}(D M)$, gives the result.

\section{Quantum solutions.}

A. The CAR. For any Cauchy surface $\Sigma$ we define an inner product on the space $C_{0}^{\infty}(D \Sigma)$ of spinors on $\Sigma$ by

$$
\left(f_{1}, f_{2}\right)=\left\langle f_{1}^{+}, \mathfrak{x} f_{2}\right\rangle=\left\langle\left(\mathfrak{x} f_{1}\right)^{+}, f_{2}\right\rangle
$$


where $\mathrm{n}$ is the forward normal to $\Sigma$. The proof that this is positive definite follows that of Proposition 1.1. Let $\mathcal{H}_{\Sigma}$ be the completion of $C_{0}^{\infty}(D \Sigma)$ in this norm, and let $\mathcal{H}_{\Sigma}^{\prime}$ be the dual Hilbert space. One can show that elements of $C_{0}^{\infty}\left(D^{*} \Sigma\right)$ regarded as distributions define elements of $\mathcal{H}_{\Sigma}^{\prime}$ and that such elements form a dense subspace in $\mathcal{H}_{\Sigma}^{\prime}$.

A representation of the CAR over $\Sigma$ is a representation of the CAR over the pair $\mathcal{H}_{\Sigma}, \mathcal{H}_{\Sigma}^{\prime}$. It consists of a Hilbert space $\mathcal{H}$ and continuous linear functions $\chi$ : $\mathcal{H}_{\Sigma}^{\prime} \rightarrow B(\mathcal{H})$ (the bounded operators on $\mathcal{H}$ ) and $\chi^{*}: \mathcal{H}_{\Sigma} \rightarrow B(\mathcal{H})$ such that

$$
\chi^{*}(f)=[\chi((f, \cdot))]^{*}
$$

and such that the anticommutator satisfies $\left\{\chi(h), \chi^{*}(f)\right\}=\langle h, f\rangle$ with all other anticommutators equal to zero. (This formulation in terms of the dual Hilbert space is due to Wightman [24].) Note that if $f \in C_{0}^{\infty}(D \Sigma)$, then $(f, \cdot) \in \mathcal{H}_{\Sigma}^{\prime}$ is just $(x f)^{+}$so we have $\chi^{*}(f)=\left[\chi\left((x f)^{+}\right)\right]^{*}$.

EXAMPLE (Fock REPRESENTATION). Let $\mathcal{H}^{0}=\mathbf{C}, \mathcal{H}^{n}=\Lambda^{n}\left(\mathcal{H}_{\Sigma}\right)$ and $\mathcal{H}=$ $\bigoplus_{n=0}^{\infty} \mathcal{H}^{n}$. We define $\chi^{*}, \chi$ as creation and annihilation operators. If $\phi=$ $\left(\phi_{0}, \phi_{1}, \phi_{2}, \ldots\right) \in \mathcal{H}$ then

$$
(\chi(h) \phi)_{n}=\sqrt{n+1}\left\langle h, \phi_{n+1}\right\rangle, \quad\left(\chi^{*}(f) \phi\right)_{n}=\sqrt{n} f \wedge \phi_{n-1}
$$

where $\left\langle h, \phi_{n+1}\right\rangle$ means evaluation on the first factor.

Note that for Minkowski space this Fock representation is different from the usual Fock representation in which $\chi$ is the sum of an annihilation operator for particles and a creation operator for antiparticles.

B. The field operators. We now construct field operators satisfying the Dirac equation using any representation $\chi$ of the CAR as data. If we think of $\chi$ as an (operator-valued) distribution cross-section of $D \Sigma$, then by analogy with the classical problem we should define $\psi=-i S \rho^{\prime} \not \chi$. More precisely, for $h \in$ $C_{0}^{\infty}\left(D^{*} M\right)$ we define

$$
\psi(h)=i \chi(x \rho S h) .
$$

Note that $\operatorname{supp}(\rho S h)$ is compact $\left(J^{ \pm}(K) \cap \Sigma\right.$ is compact for compact $K$, Cauchy surface $\Sigma$ ) so that $\not x \rho S h \in C_{0}^{\infty}\left(D^{*} \Sigma\right) \subset \mathcal{F}_{\Sigma}^{\prime}$. The field satisfies $(-i \nabla+m) \psi=0$ in the sense of distributions (i.e. $\psi((i \nabla+m) h)=0$ ) since $S(i \nabla+m)=0$ on $C_{0}^{\infty}\left(D^{*} M\right)$. If we also define, for $f \in C_{0}^{\infty}(D M), \psi^{+}(f)=\left[\psi\left(f^{+}\right)\right]^{*}$ then $\psi^{+}$is linear in $f$ and satisfies $(i \nabla+m) \psi^{+}=0$. One can check that $\psi^{+}$can be expressed as $\psi^{+}(f)=-i \chi^{*}(\rho S f)$. The anticommutator can now be computed to be

$$
\left\{\psi(h), \psi^{+}(f)\right\}=\langle x \rho S h, \rho S f\rangle=-i\langle h, S f\rangle
$$

where we use Proposition 2.4(d). (This form for the anticommutator was anticipated by Lichnerowicz [16].)

If $\hat{\Sigma}$ is another Cauchy surface then the restriction of $\psi$ to $\hat{\Sigma}$ is given by $\hat{\chi}=\hat{\rho} \psi=-i \hat{\rho} S \rho^{\prime} \not \chi \chi$. This means, for $h \in C_{0}^{\infty}\left(D^{*} \hat{\Sigma}\right), \hat{\chi}(h)=i \chi\left(\not \rho \rho \hat{\rho}^{\prime} h\right)$. We also define for $f \in C_{0}^{\infty}(D \hat{\Sigma})$

$$
\hat{\chi}^{*}(f) \equiv\left[\hat{\chi}\left((\hat{\mathrm{x}} f)^{+}\right)\right]^{*}=-i \chi^{*}\left(\rho S \hat{\rho}^{\prime} \hat{\mathrm{x}} f\right) .
$$


These expressions make sense since $\rho S \hat{\rho}^{\prime}$ maps $C_{0}^{\infty}(D \hat{\Sigma})$ (or $C_{0}^{\infty}\left(D^{*} \hat{\Sigma}\right)$ ) to $C_{0}^{\infty}(D \Sigma)$ (or $C_{0}^{\infty}\left(D^{*} \Sigma\right)$ ). For the anticommutator we have

$$
\left\{\hat{\chi}(h), \hat{\chi}^{*}(f)\right\}=\left\langle\rho S \hat{\rho}^{\prime} h, \not \mathfrak{x} \rho S \hat{\rho}^{\prime} \hat{x} f\right\rangle .
$$

By the identity (1.7) we have that, for solutions $u, v$ with compact support on $\Sigma$, $\langle\rho v, \not x \rho u\rangle=\langle\hat{\rho} v, \hat{x} \hat{\rho} u\rangle$. Making this replacement above and using Proposition 2.4(c) the anticommutator is just $\langle h, f\rangle$. Thus restriction to another surface gives a representation of the CAR over that surface. Note that if $\Sigma=\hat{\Sigma}$ then $\hat{\chi}=\chi$ by the adjoint of Proposition 2.4(c).

We summarize our results for this section:

THEOREM 3.1. Let $(M, g, S M, p)$ be a globally hyperbolic manifold with spin structure. Let $(\Sigma, \chi)$ be a representation of the CAR over a Cauchy surface. Then $\psi=-i S \rho^{\prime} \not \chi \chi$ is a solution of the Dirac equation with data $\chi$ on $\Sigma$, and with anticommutator $\left\{\psi(h), \psi^{+}(f)\right\}=i^{-1}\langle h, S f\rangle$.

\section{Local algebras.}

A. Definitions. Let $\psi$ be the field operator defined in the previous theorem. We now define for any open set $\mathcal{O} \subset M$ local field algebras by $\mathscr{F}(\mathcal{O}) \equiv C^{*}$ algebra generated by $\left\{\psi(h): h \in C_{0}^{\infty}\left(D^{*} M\right)\right.$, supp $\left.h \subset \mathcal{O}\right\}$. These are all subalgebras of $\mathscr{F}=\mathscr{F}(M)$. Another characterization of $\mathscr{F}$ is $\mathscr{F}=C^{*}$ algebra generated by $\{\chi(h)$ : $\left.h \in \mathcal{F}_{\Sigma}^{\prime}\right\}$ or $\left\{\chi(h): h \in C_{0}^{\infty}\left(D^{*} \Sigma\right)\right\}$. Indeed if the latter algebra is denoted $\hat{\mathscr{F}}$, then $\mathscr{F} \subset \hat{\mathscr{F}}$ by the definition $\psi(h)=i \chi(\chi \rho S h)$. On the other hand for any $h_{1} \in$ $C_{0}^{\infty}\left(D^{*} \Sigma\right)$ there exists $h \in C_{0}^{\infty}\left(D^{*} M\right)$ such that $i \not x \rho S h=h_{1}$ (see [6, Lemma A.3]). Then we have $\chi\left(h_{1}\right)=\psi(h)$ which implies $\hat{\mathscr{F}} \subset \mathscr{F}$ and hence $\hat{\mathscr{F}}=\mathscr{F}$.

The field algebras are not suitable as an algebra of observables since the algebras for causally independent regions do not commute. We remedy this defect by passing to subalgebras. There is some arbitrariness in the choice of the subalgebras, but for definiteness we make the following choice. The algebra of observables for $\vartheta \subset M$ is

$$
\mathcal{Q}(\mathcal{O})=C^{*} \text { algebra generated by }\left[\psi^{+}(f) \psi(h)\right], \quad \operatorname{supp} f, \operatorname{supp} h \subset \mathcal{O}
$$

and the full algebra is $Q=Q(M)$.

Before proceeding with our study of these algebras we mention some related literature which the reader may wish to consult. A slightly different algebraic formulation for field theory on manifolds, also applicable to the Dirac field, has been given by Isham [13], Kay [15], and Hajicek [12]. Bongaarts [2] gives an algebraic treatment of the Dirac field on Minkowski space with external potentials. The relation between the field algebras and algebras of observables on Minkowski space has been studied in great generality by Doplicher, Haag, and Roberts [7]. For the general philosophy of the algebraic approach there is Haag-Kastler [10] or Segal [21].

B. Categories. We want to explore the circumstances under which the algebraic structures we have defined are equivalent. To accomplish this it is convenient to use the language of category theory. We introduce the following categories. 
$C^{*}$ Nets. An object is a collection of $C^{*}$ algebras $\{A(i)\}_{i \subset I}$ indexed by a collection of subsets of a given set $I$ such that $A(i) \subset A\left(i^{\prime}\right)$ whenever $i \subset i^{\prime}$. The index set includes $I$ so $A_{i} \subset A_{I}$ for all $i$. A morphism from $\left\{A_{1}(i)\right\}_{i \subset I_{1}}$ to $\left\{A_{2}(i)\right\}_{i \subset I_{2}}$ is a $C^{*}$-algebra isomorphism $\alpha: A_{I_{1}} \rightarrow A_{I_{2}}$ and a bijection $f: I_{1} \rightarrow I_{2}$ such that $\alpha\left[A_{1}(i)\right]=A_{2}(f[i])$ for all $i \subset I_{1}$. Morphisms are composed by $\left(\alpha_{2}, f_{2}\right) \circ\left(\alpha_{1}, f_{1}\right)=\left(\alpha_{2} \circ \alpha_{1}, f_{2} \circ f_{1}\right)$. A morphism in this category will be called a net isomorphism.

Note that $\{\mathscr{F}(\theta)\}_{\Theta \subset M}$ and $\{\mathscr{Q}(\vartheta)\}_{\Theta \subset M}$ are objects in this category indexed by the open subsets $\theta \subset M$. We abbreviate them as $\mathscr{F}(\cdot)$ and $\mathcal{Q}(\cdot)$.

Lor. An object is a Lorentzian manifold $(M, g)$ (always globally hyperbolic). A morphism from $\left(M_{1}, g_{1}\right)$ to $\left(M_{2}, g_{2}\right)$ is a diffeomorphism $\kappa: M_{1} \rightarrow M_{2}$ such that $g_{1}=\kappa^{*}\left(g_{2}\right)$, i.e. a morphism is an isometry.

LorSpin. An object $m=(M, g, S M, p)$ is a Lorentzian manifold $(M, g)$ with spin

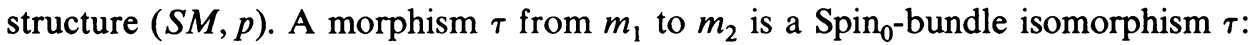
$S_{1} M_{1} \rightarrow S_{2} M_{2}$ such that the induced map $\bar{\tau}: M_{1} \rightarrow M_{2}$ of the base spaces is an isometry and such that the following diagram is commutative:

$$
\begin{array}{ccc}
S_{1} M_{1} & \stackrel{\tau}{\rightarrow} & S_{2} M_{2} \\
\downarrow p_{1} & & \downarrow p_{2} \\
F M_{1} & \stackrel{\tau_{F}}{\rightarrow} & F M_{2}
\end{array}
$$

where $\tau_{F}$ is the $\mathscr{L}_{0}$-bundle isomorphism induced on the orthonormal frame bundles by $\bar{\tau}$. We call such a morphism $\tau$ a spin structure isomorphism. Note that if $M_{1}=M_{2}$ and $\bar{\tau}=$ id then $\tau$ is an equivalence by our earlier definition,

LorSpinRep. An object is a pair $(m, r)$ where $m$ is an object in LorSpin and $r=(\Sigma, \chi)$ is a representation $\chi$ of the CAR over a Cauchy surface $\Sigma$ in $M$. A morphism from $\left(m_{1}, r_{1}\right)$ to $\left(m_{2}, r_{2}\right)$ is just a morphism $\tau: m_{1} \rightarrow m_{2}$ in LorSpin which we write as $\left(\tau, r_{2}, r_{1}\right)$. Then the identity is (id, $\left.r, r\right)$ and the law of composition for $\tau_{1}: m_{1} \rightarrow m_{2}$ and $\tau_{2}: m_{2} \rightarrow m_{3}$ is

$$
\left(\tau_{2}, r_{3}, r_{2}\right) \circ\left(\tau_{1}, r_{2}, r_{1}\right)=\left(\tau_{2} \circ \tau_{1}, r_{3}, r_{1}\right) \text {. }
$$

C. Functors. For each choice $(m, r)$ of manifold with spin structure $m=$ $(M, g, S M, p)$ and representation $r=(\Sigma, \chi)$ we let $\psi_{m, r}$ be the field operator and $\mathscr{F}_{m, r}, \mathscr{F}_{m, r}(\Theta), \mathcal{Q}_{m, r}, \mathcal{Q}_{m, r}(\Theta)$ be the associated algebras.

THEOREM 4.1. For any spin structure isomorphism $\tau: m_{1} \rightarrow m_{2}$ and any representations $r_{1}$ on $m_{1}$ and $r_{2}$ on $m_{2}$ there exists a unique isomorphism $\alpha\left(\tau, r_{2}, r_{1}\right)$ from $\mathscr{F}_{m_{1}, r_{1}}$ to $\mathscr{F}_{m_{2}, r_{2}}$ such that

$$
\alpha\left(\tau ; r_{2}, r_{1}\right)\left(\psi_{m_{1}, r_{1}}(h)\right)=\psi_{m_{2}, r_{2}}\left(\hat{\tau}_{*} h\right)
$$

where $\hat{\tau}: D_{1}^{*} M_{1} \rightarrow D_{2}^{*} M_{2}$ is the induced mapping on spinor bundles.

Proof. We shorten the notation to $\psi_{j}=\psi_{m_{j}, r_{j}}$ and $\mathscr{F}_{j}=\mathscr{F}_{m_{j}, r_{j}}, j=1,2$. The proof comes in three parts.

Part I. We consider the case $m_{1}=m_{2}=m$ and $\tau=$ id. At first suppose also that $\Sigma_{1}=\Sigma_{2}=\Sigma$, but that $\chi_{1}$ and $\chi_{2}$ are distinct representations of the CAR over $\mathcal{H}_{\Sigma}$. 
By a general theorem (see for example Slawny [22]) $\chi_{1}$ and $\chi_{2}$ generate the same $C^{*}$ algebra. That is there is an isomorphism $\alpha: \mathscr{F}_{1} \rightarrow \mathscr{F}_{2}$ such that $\alpha\left(\chi_{1}(h)\right)=\chi_{2}(h)$.

Now consider the general case $\Sigma_{1} \neq \Sigma_{2}, \chi_{1} \neq \chi_{2}$. Let $T_{21}: C_{0}^{\infty}\left(D^{*} \Sigma_{1}\right) \rightarrow$ $C_{0}^{\infty}\left(D^{*} \Sigma_{2}\right)$ be defined by $T_{21}=i \not x_{2} \rho_{2} S \rho_{1}^{\prime}$. According to the discussion in $\S I I I$, $\tilde{\chi}_{1}(h)=\chi_{2}\left(T_{21} h\right)$ also defines a representation of the CAR over $\Sigma_{1}$, and since also $\chi_{2}(k)=\tilde{\chi}_{1}\left(T_{12} k\right)$ we have $\tilde{\mathscr{F}}_{1}=\tilde{F}_{2}$. Let $\alpha$ be the isomorphism from $\mathscr{F}_{1}$ to $\tilde{\mathscr{F}}_{1}$ such that $\alpha\left(\chi_{1}(h)\right)=\tilde{\chi}_{1}(h)$, as above. Then $\alpha: \mathscr{F}_{1} \rightarrow \mathscr{F}_{2}$ and $\alpha\left(\chi_{1}(h)\right)=\chi_{2}\left(T_{21} h\right)$. Finally by the adjoint of Proposition 2.4(d) we have

$$
\begin{aligned}
\alpha\left(\psi_{1}(h)\right) & =\alpha\left(i \chi_{1}\left(\mathfrak{x}_{1} \rho_{1} S h\right)\right)=\chi_{2}\left(-\mathfrak{K}_{2} \rho_{2} S \rho_{1}^{\prime} \mathfrak{K}_{1} \rho_{1} S h\right) \\
& =i \chi_{2}\left(\mathfrak{x}_{2} \rho_{2} S h\right)=\psi_{2}(h) .
\end{aligned}
$$

Thus we have constructed $\alpha$ (id, $\left.r_{2}, r_{1}\right)$.

Part II. We now digress to develop some facts about spin-structure isomorphisms $\tau: m_{1} \rightarrow m_{2}$. The $\mathrm{Spin}_{0}$-bundle isomorphism $\tau: S_{1} M_{1} \rightarrow S_{2} M_{2}$ induces a vector bundle isomorphism $\hat{\tau}: D_{1} M_{1} \rightarrow D_{2} M_{2}$ with the property that if $E_{1}, E_{2}$ are crosssections of $S_{1} M_{1}, S_{2} M_{2}$ such that $\tau_{*}\left(E_{1}\right)=E_{2}$, then the associated moving frames $E_{1, A}, E_{2, A}$ in $D_{1} M_{1}, D_{2} M_{2}$ satisfy $\hat{\tau}_{*}\left(E_{1, A}\right)=E_{2, A}$. Here $\tau_{*}, \hat{\tau}_{*}$ are the induced maps on cross-sections defined by $\tau_{*}(E)=\tau \circ E \circ \bar{\tau}^{-1}$ and $\hat{\tau}_{*}(f)=\hat{\tau} \circ f \circ \bar{\tau}^{-1}$. We have the general transformation law for spinors

$$
\hat{\tau}_{*}\left(f^{A} E_{1, A}\right)=\left(f^{A} \circ \bar{\tau}^{-1}\right) E_{2, A} .
$$

The isomorphism $\tau$ also induces a vector bundle isomorphism $\hat{\tau}: T M_{1} \rightarrow T M_{2}$ by $\hat{\tau} \equiv D \bar{\tau}$, and this induces a map $\hat{\tau}_{*}$ on vector fields. If $e_{1}=p_{1} \circ E_{1}$ and $e_{2}=$ $p_{2} \circ E_{1}$ are the orthonormal frames associated with $E_{1}, E_{2}$ as above then $\hat{\tau}_{*} e_{1, a}=$ $e_{2, a}$. To see this note first that $\left(\tau_{F} \xi\right)_{a}=\hat{\tau} \xi_{a}$ by definition so that $\hat{\tau}_{*} e_{1, a}=\left(\left(\tau_{F}\right)_{*} e_{1}\right)_{a}$. The result then follows by

$$
\left(\tau_{F}\right)_{*} e_{1}=\tau_{F} \circ p_{1} \circ E_{1} \circ \bar{\tau}^{-1}=p_{2} \circ \tau \circ E_{1} \circ \bar{\tau}^{-1}=p_{2} \circ E_{2}=e_{2}
$$

where we use the definition of $\tau$.

The maps $\hat{\tau}$ on $D_{1} M_{1}$ and $T M_{1}$ induce a map $\hat{\tau}$ on the full spinor-tensor algebra which commutes with contractions and tensor products. In terms of bases determined by $E_{1}$ and $E_{2}=\tau_{*}\left(E_{1}\right)$ we have for example $\hat{\tau}_{*}\left(f_{a B}^{A} e_{1}^{a} \otimes E_{1, A} \otimes E_{1}^{B}\right)=$ $\left(f_{a B}^{A} \circ \bar{\tau}^{-1}\right) e_{2}^{a} \otimes E_{2, A} \otimes E_{2}^{B}$. Note in particular that $\hat{\tau}_{*} \gamma_{1}=\gamma_{2}$.

Next we examine the effect of $\tau$ on connections. Let $\tau^{*}, \tau_{F}^{*}$ be the pull-back operation on one-forms on $S_{2} M_{2}, F M_{2}$ (not the pull-back of cross-sections). The connection forms are related by $\tau_{F}^{*} \omega_{2}=\omega_{1}$ and thus using $p_{2} \circ \tau=\tau_{F} \circ p_{1}$ we have

$$
\tau^{*} \sigma_{2}=\left(\Lambda^{\prime}\right)^{-1} \tau^{*} p_{2}^{*} \omega_{2}=\left(\Lambda^{\prime}\right)^{-1} p_{1}^{*} \tau_{F}^{*} \omega_{2}=\sigma_{1}
$$

Since $E_{2} \circ \bar{\tau}=\tau \circ E_{1}$ it follows that $\bar{\tau}^{*}\left(E_{2}^{*} \sigma_{2}\right)=E_{1}^{*} \sigma_{1}$. Now $\left(\bar{\tau}^{-1}\right)^{*}$ (the pull-back of one-forms) can also be written as $\hat{\tau}_{*}$ (the push-forward of sections) in our notation. Thus we have $\hat{\tau}_{*}\left(\sigma_{1 B}^{A}\right)=\sigma_{2 B}^{A}$ and hence

$$
\hat{\tau}_{*}\left(\nabla_{1} E_{1, A}\right)=\hat{\tau}_{*}\left(\sigma_{1 A}^{B} \otimes E_{1, B}\right)=\sigma_{2 A}^{B} \otimes E_{2, B}=\nabla_{2} E_{2, A} \text {. }
$$

This shows that $\hat{\tau}_{*} \nabla_{1}=\nabla_{2} \hat{\tau}_{*}$ and since $\hat{\tau}_{*} \gamma_{1}=\gamma_{2}$ we have that $\hat{\tau}_{*}\left(-i \nabla_{1}+m\right)=$ $\left(-i \nabla_{2}+m\right) \hat{\tau}_{*}$. By the uniqueness of fundamental solutions:

$$
\hat{\tau}_{*} S_{1}^{ \pm}=S_{2}^{ \pm} \hat{\tau}_{*}
$$


Part III. Now we consider the general case $m_{1} \neq m_{2}, r_{1} \neq r_{2}, \tau: m_{1} \rightarrow m_{2}$. Let $\tilde{\Sigma}_{1}=\bar{\tau}^{-1}\left(\Sigma_{2}\right)$ and define $\hat{\tau}_{*}: C_{0}^{\infty}\left(D_{1}^{*} \tilde{\Sigma}_{1}\right) \rightarrow C_{0}^{\infty}\left(D_{2}^{*} \Sigma_{2}\right)$ to be the push-forward of cross-sections by $\hat{\tau}_{*} h=\hat{\tau} \circ h \circ \bar{\tau}^{-1}$. We define a representation $\tilde{r}_{1}=\left(\tilde{\Sigma}_{1}, \tilde{\chi}_{1}\right)$ for $m_{1}$ by $\tilde{\chi}_{1}(h)=\chi_{2}\left(\hat{\tau}_{*} h\right)$. This together with $\tilde{\chi}_{1}^{*}(f)=\chi_{2}^{*}\left(\hat{\tau}_{*} f\right)$ forms a representation of the CAR. Indeed the anticommutator is

$$
\left\langle\hat{\tau}_{*} h, \hat{\tau}_{*} f\right\rangle=\int_{M_{2}}(h f) \circ \bar{\tau}^{-1} d V_{2}=\int_{M_{1}} h f d V_{1}=\langle h, f\rangle .
$$

Furthermore $\hat{\tau}_{*} \tilde{\mathbb{X}}_{1}=\not \mathbb{X}_{2}$ and $\left(\hat{\tau}_{*} f\right)^{+}=\hat{\tau}_{*} f^{+}$and so

$$
\tilde{\chi}_{1}^{*}(f)=\chi_{2}\left(\left(\not_{2} \hat{\tau}_{*} f\right)^{+}\right)^{*}=\chi_{2}\left(\hat{\tau}_{*}\left(\tilde{x}_{1} f\right)^{+}\right) *=\tilde{\chi}_{1}\left(\left(\tilde{\mathbb{x}}_{1} f\right)^{+}\right)^{*}
$$

as required. Since the transformation $\chi_{2} \rightarrow \tilde{\chi}_{1}$ is invertible we have $\tilde{\mathscr{F}}_{1}=\mathscr{F}_{2}$. We also have

$$
\begin{aligned}
\tilde{\psi}_{1}(h) & =i \tilde{\chi}_{1}\left(\tilde{\mathbb{X}}_{1} \tilde{\rho}_{1} S_{1} h\right)=i \chi_{2}\left(\hat{\tau}_{*} \tilde{\mathrm{p}}_{1} \tilde{\rho}_{1} S_{1} h\right) \\
& =i \chi_{2}\left(\not_{2} \rho_{2} S_{2} \hat{\tau}_{*} h\right)=\psi_{2}\left(\hat{\tau}_{*} h\right) .
\end{aligned}
$$

Here we use $\hat{\tau}_{*} S_{1}=S_{2} \hat{\tau}_{*}$ from Part II.

Now let $\alpha=\alpha\left(\mathrm{id}, \tilde{r}_{1}, r_{1}\right)$ be the isomorphism from Part I. Then $\alpha: \tilde{F}_{1} \rightarrow \tilde{\mathscr{F}}_{1}$ and $\alpha\left(\psi_{1}(h)\right)=\tilde{\psi}_{1}(h)$. From above we see that $\alpha: \mathscr{F}_{1} \rightarrow \mathscr{F}_{2}$ and $\alpha\left(\psi_{1}(h)\right)=\psi_{2}\left(\hat{\tau}_{*} h\right)$. Thus $\alpha$ is the required $\alpha\left(\tau, r_{2}, r_{1}\right)$. Uniqueness follows since $\alpha$ is determined by its action on the dense set of polynomials in $\psi$.

REMARKS. (1) Since $\hat{\tau}_{*}$ maps the sections of $D_{1}^{*} M_{1}$ with support in $\theta$ onto the sections of $D_{2}^{*} M_{2}$ with support in $\bar{\tau}(\Theta)$, we have that $\alpha\left(\tau, r_{2}, r_{1}\right)$ maps the polynomials in $\mathscr{F}_{m_{1}, r_{1}}(\theta)$ onto the polynomials in $\mathscr{F}_{m_{2}, r_{2}}(\bar{\tau}(\theta))$, and hence that

$$
\alpha\left(\tau, r_{2}, r_{1}\right)\left[\mathscr{F}_{m_{1}, r_{1}}(\theta)\right]=\mathscr{F}_{m_{2}, r_{2}}(\bar{\tau}(\theta))
$$

This says that $\alpha\left(\tau, r_{2}, r_{1}\right)$ and $\bar{\tau}$ define a net isomorphism from $\mathscr{F}_{m_{1}, r_{1}(\cdot)}$ to $\mathscr{F}_{m_{2}, r_{2}(\cdot)}$.

(2) From $\psi^{+}(f)=\left[\psi\left(f^{+}\right)\right]^{*}$ we obtain that $\alpha\left(\tau, r_{2}, r_{1}\right)\left[\psi_{m_{1}, r_{1}}^{+}(f)\right]=\psi_{m_{2}, r_{2}}^{+}\left(\hat{\tau}_{*} f\right)$ and this gives

$$
\alpha\left(\tau, r_{2}, r_{1}\right)\left[\mathbb{Q}_{m_{1}, r_{1}}(\theta)\right]=\mathbb{Q}_{m_{2}, r_{2}}(\bar{\tau}(\theta))
$$

Thus we have a net isomorphism from $\mathbb{Q}_{m_{1}, r_{1}}(\cdot)$ to $\mathbb{Q}_{m_{2}, r_{2}(\cdot)}$.

(3) If $\tau=$ id then $\hat{\tau}_{*}=\mathrm{id}$ and also $\hat{\tau}_{2 *} \circ \hat{\tau}_{1 *}=\left(\tau_{2} \circ \tau_{1}\right)^{\wedge}$. This enables us to show from the action of $\alpha$ on $\psi$ that the identities

$$
\begin{aligned}
\alpha(\mathrm{id}, r, r) & =\mathrm{id}, \\
\alpha\left(\tau_{2}, r_{3}, r_{2}\right) \alpha\left(\tau_{1}, r_{2}, r_{1}\right) & =\alpha\left(\tau_{2} \circ \tau_{1}, r_{3}, r_{1}\right)
\end{aligned}
$$

hold on polynomials in $\psi$ and hence on the full algebras. Similar identities hold for the net isomorphisms $\left(\alpha\left(\tau, r_{2}, r_{1}\right), \bar{\tau}\right)$.

Thus we have proved the following result:

THEOREM 4.2. The maps $(m, r) \rightarrow \mathscr{F}_{m, r}(\cdot)$ and $(m, r) \rightarrow \mathbb{Q}_{m, r}(\cdot)$ together with $\left(\tau, r_{2}, r_{1}\right) \rightarrow\left(\alpha\left(\tau, r_{2}, r_{1}\right), \bar{\tau}\right)$ define functors from LorSpinRep to $C^{*}$ Nets.

This theorem makes precise the sense in which our algebraic structure does not depend on special choices we have made. In particular specializing to $\bar{\tau}=\mathrm{id}$ it says 
that the nets do not depend on equivalent spin structures, Cauchy surfaces, or CAR representations. We also give another version of this result which singles out a particular representation for each $m$.

Let $r=f(m)$ be a choice function assigning representations to manifolds. We define

$$
\mathbb{Q}_{m}^{f}(\cdot)=\mathbb{Q}_{m, f(m)}(\cdot)
$$

and for $\tau: m_{1} \rightarrow m_{2}$ we define $\alpha^{f}(\tau): \mathbb{Q}_{m_{1}}^{f} \rightarrow \mathbb{Q}_{m_{2}}^{f}$ by $\alpha^{f}(\tau)=\alpha\left(\tau, f\left(m_{2}\right), f\left(m_{1}\right)\right)$.

THEOREM 4.3. (a) For any choice function $f$, the map $m \rightarrow \mathbb{Q}_{m}^{f}(\cdot)$ together with $\tau \rightarrow\left(\alpha^{f}(\tau), \bar{\tau}\right)$ defines a functor from LorSpin to $C^{*}$ Nets.

(b) Different choice functions give naturally equivalent functors.

Proof. (a) is straightforward. For (b) let $f, g$ be two choice functions. The assertion is that for every $m$ there is a net isomorphism $\beta_{m}$ from $\mathbb{Q}_{m}^{f}(\cdot)$ to $\mathbb{Q}_{m}^{g}(\cdot)$ such that the following diagram commutes for each $\tau: m_{1} \rightarrow m_{2}$ :

$$
\begin{array}{ccc}
\mathbb{Q}_{m_{1}}^{f}(\cdot) & \stackrel{\beta_{m_{1}}}{\rightarrow} & \mathbb{Q}_{m_{1}}^{g}(\cdot) \\
\left(\alpha^{f}(\tau), \bar{\tau}\right) \downarrow & & \downarrow\left(\alpha^{8}(\tau), \bar{\tau}\right) \\
\mathbb{Q}_{m_{2}}^{f}(\cdot) & \stackrel{\beta_{m_{2}}}{\rightarrow} & \mathbb{Q}_{m_{2}}^{g}(\cdot)
\end{array}
$$

This is fulfilled by taking $\beta_{m}=(\alpha(\mathrm{id}, g(m), f(m))$, id).

REMARK. For the algebra of observables it is possible to continue and make choices of spin structure for each manifold to obtain a family of equivalent functors from Lor to $C^{*}$ Nets. We do not include details.

D. Axioms. We now collect the most important properties of our algebras. These are properties which might be expected to hold for any quantum field theory on a Lorentzian manifold (not necessarily globally hyperbolic). They are the generalized Haag-Kastler axioms [6], [10].

THEOREM 4.4. $\mathcal{Q}(\cdot)=\mathbb{Q}_{m, r}(\cdot)$ satisfies the following properties:

(1) $Q(\cdot)$ is an increasing net of $C^{*}$ algebras indexed by a collection of open sets $\theta \subset M$.

(2) $Q=Q(M)$ is primitive.

(3) If $\Theta, \Theta^{\prime}$ are causally independent then $\left[\mathscr{Q}(\theta), \mathcal{Q}\left(\theta^{\prime}\right)\right]=0$.

(4) If $\theta$ is causally dependent on $\theta^{\prime}$ then $\mathbb{Q}(\theta) \subset \mathbb{Q}\left(\theta^{\prime}\right)$.

(5) For any isomorphism between space-time structures there is a functorial isomorphism between the associated algebraic structures.

Proof. (1) is trivial. (2) follows since $C^{*}$ algebras generated by the CAR are primitive. For (3) we compute

$$
\left[\psi^{+}\left(f_{1}\right) \psi\left(h_{1}\right), \psi^{+}\left(f_{2}\right) \psi\left(h_{2}\right)\right]=-i\left\langle h_{1}, S f_{2}\right\rangle \psi^{+}\left(f_{1}\right) \psi\left(h_{2}\right)+i\left\langle h_{2}, S f_{1}\right\rangle \psi^{+}\left(f_{2}\right) \psi\left(h_{1}\right) \text {. }
$$

Let $f_{j}, h_{j}$ have support in $\theta_{j}, j=1,2$, and suppose that $\theta_{1}, \theta_{2}$ are causally independent, i.e. there is no causal curve joining a point in $\theta_{1}$ to a point in $\theta_{2}$. By the support properties of $S$ we have $\left\langle h_{1}, S f_{2}\right\rangle=\left\langle h_{2}, S f_{1}\right\rangle=0$ so the above 
commutator vanishes. It follows that $\left[\mathscr{Q}\left(\theta_{1}\right), \mathbb{Q}\left(\vartheta_{2}\right)\right]=0$. A weak form of (4) is proved in [6] for the scalar field and the same proof works here. Finally we regard (5) as being fulfilled by Theorem 4.3(a).

\section{REFERENCES}

1. S. Avis and C. Isham, Quantum field theory and fibre bundles in a general space-time, Recent Developments in Gravitation (Levy and Deser, eds.), Plenum Press, New York, 1979.

2. P. Bongaarts, The electron-positron field coupled to external electromagnetic potentials as an elementary $C^{*}$-algebra theory, Ann. Physics 56 (1970), 108-139.

3. Y. Choquet-Bruhat, Hyperbolic differential equations on a manifold, Battelle Rencontres (DeWitt and Wheeler, eds.), Benjamin, New York, 1968.

4. Y. Choquet-Bruhat, C. DeWitt-Morette and M. Dillard-Bleick, Analysis, manifolds, and physics, North-Holland, New York, 1977.

5. B. Dewitt, C. F. Hart and C. J. Isham, Topology and quantum field theory, preprint.

6. J. Dimock, Algebras of local observables on a manifold, Comm. Math. Phys. 77 (1980), 219-228.

7. S. Doplicher, R. Haag and J. Roberts, Fields, observables, and gauge transformations. I, II, Comm. Math. Phys. 13 (1969), 1; 15 (1969), 173.

8. R. Geroch, Spinor structure of space-times in general relativity. I, II, J. Math. Phys. 9 (1968), 1739; 11 (1970), 343.

9. $\longrightarrow$ The domain of dependence, J. Math. Phys. 11 (1970), 437.

10. R. Haag and D. Kastler, An algebraic approach to quantum field theory, J. Math. Phys. 5 (1964), 848.

11. S. Hawking and G. Ellis, The large scale structure of space-time, Cambridge Univ. Press, Cambridge, 1973.

12. P. Hajicek, Observables for quantum fields on curved backgrounds, Lecture Notes in Math., vol. 676, Springer-Verlag, Berlin, 1978, pp. 535-566.

13. C. Isham, Quantum field theory in curved space-time, Lecture Notes in Math., vol. 676, SpringerVerlag, Berlin, 1978, pp. 495-512.

14. __ Twisted quantum fields in a curved space-time, Proc. Roy. Soc. London Ser. A 362 (1978), 383.

15. B. Kay, Linear spin-zero quantum fields in external gravitational and scalar fields. I, II, Comm. Math. Phys. 62 (1978), 55; 71 (1980), 29.

16. A. Lichnerowicz, Champs spinoriel et propagateurs, Bull. Soc. Math. France 92 (1964), 11.

17. _ Topics on space-times, Battelle Recontres (DeWitt and Wheeler, eds.), Benjamin, New York, 1968.

18. J. Leray, Hyperbolic differential equations, lecture notes, Princeton, 1953.

19. W. Pauli, Contributions mathématiques à la théorie des matrices de Dirac, Ann. Inst. H. Poincaré VI (1963), 8.

20. H. Petry, Exotic spinors in superconductivity, J. Math. Phys. 20 (1979), 231.

21. I. Segal, Foundations of the theory of dynamical systems of infinitely many degrees of freedom. I, Mat.-Fys. Medd. Danske Vid. Selsk. 31 (1959), 121.

22. J. Slawny, On factor representations and the $C^{*}$-algebra of the canonical commutation relations, Comm. Math. Phys. 24 (1972), 151.

23. A. Wightman, The Dirac equation, Aspects of Quantum Theory (Salam and Wigner, eds.), Cambridge Univ. Press, Cambridge, 1972, pp. 109.

24. __ Relativistic wave equations as singular hyperbolic systems, Proc. Sympos. Pure Math., vol. 23, Amer. Math. Soc., Providence, R. I., 1973.

School of Natural Sciences, Institute for Advanced Study, Princeton, New Jersey 08540

Current address: Department of Mathematics, SUNY at Buffalo, Buffalo, New York 14214 